\title{
LEVEL SETS AND CONTINUITY OF CONJUGATE CONVEX FUNCTIONS
}

\author{
BY \\ R. T. ROCKAFELLAR(1)
}

1. Introduction. A proper convex function on a vector space $F$ over the real numbers $R$ is an everywhere-defined function $f$ with values in $(-\infty,+\infty]$, not identically $+\infty$, such that

$$
f\left(\lambda x_{1}+(1-\lambda) x_{2}\right) \leqq \lambda f\left(x_{1}\right)+(1-\lambda) f\left(x_{2}\right)
$$

for all $x_{i} \in F, x_{2} \in F, 0<\lambda<1$. Its effective domain is the nonempty convex set

$$
\operatorname{dom} f=\{x \in F \mid f(x)<\infty\} .
$$

A finite-valued convex function on a nonempty convex set $C$ in $F$ can always be extended to a proper convex function on $F$ by assigning it the value $+\infty$ outside of $C$.

Let $F$ and $G$ be real vector spaces in duality with respect to a bilinear functional $(x, y)$ for $x \in F$ and $y \in G$ (see $[1$, p. 48]). We shall henceforth assume $F$ and $G$ have each been supplied with a topology compatible with this duality $[1$, p. 67], so that each can be identified with the space of continuous linear functionals on the other. Unless explicit notice is given, all questions of closure, continuity and boundedness refer to these given topologies. The formulas

$$
\begin{aligned}
& g(y)=\sup \{(x, y)-f(x) \mid x \in F\} \text { for all } y \in G, \\
& f(x)=\sup \{(x, y)-g(y) \mid y \in G\} \text { for all } x \in F,
\end{aligned}
$$

define a one-to-one correspondence between the lower semicontinuous (1.s.c.) proper convex functions $f$ on $F$ and the 1.s.c. proper convex functions $g$ on $G$. Functions paired by (1.3a) and (1.3b) are said to be conjugate to each other. This conjugate correspondence, discovered by Fenchel [6], was extended to infinite-dimensional spaces by Moreau [9] and Brondsted [2].

It is natural to look for interesting relationships between the conjugate correspondence among convex functions and two classical correspondences, the one between convex sets and their support functions, and the polar correspondence

Received by the editors January 22, 1964 and, in revised form, July 15, 1965.

(1) Supported by Air Force Grant AFOSR-467-63 at the Computation Center, the University of Texas. 
for convex cones. Such is our motivation here. A fact which is already known, and which will be important in our investigations, is that the two classical correspondences can be treated as special examples of the conjugate correspondence in the following way.

EXAMPLE 1A. A 1.s.c. proper convex function is an indicator function, i.e. has no values other than 0 and $+\infty$, if and only if its conjugate is positively homogeneous. The functions of the first kind on $F$ are precisely of the form $f(x)=\delta_{C}(x)=\delta(x \mid C)$, where $C$ is a nonempty closed convex set and

$$
\delta(x \mid C)=0 \text { if } x \in C,(x \mid C)=\infty \text { if } x \notin C .
$$

The conjugate of $\delta_{c}$ is the support fiunction $\sigma_{c}$ of $C$, where

$$
\sigma_{c}(y)=\sigma(y \mid C)=\sup \{(x, y) \mid x \in C\} \text { for all } y \in G .
$$

Dually, the indicator functions of the nonempty closed convex sets in $G$ are the conjugates of the support functions of these sets, which are precisely the positively homogeneous 1.s.c. proper convex functions on $F$.

EXAmple 1B. According to Example 1A, a 1.s.c. proper convex function is a positively homogeneous indicator function if and only if its conjugate is also. Such functions are the indicator functions of nonempty closed convex cones (with vertex at the origin). For two such cones $K \subseteq F$ and $L \subseteq G, f=\delta_{K}$ and $g=\delta_{L}$ are conjugate to one another if and only if $K$ and $L$ are polar to one another, i.e.

$$
\begin{aligned}
& L=\{y \in G \mid(x, y) \leqq 0 \text { for all } x \in K\}, \\
& K=\{x \in F \mid(x, y) \leqq 0 \text { for all } y \in L\}
\end{aligned}
$$

In particular, the annihilator correspondence between subspaces of $F$ and $G$ may be expressed by the conjugacy of indicator functions of subspaces.

Associated with any 1.s.c. proper convex function $f$ on $F$ are various distinguished convex sets, cones, and positively homogeneous convex functions. What happens to all these objects under the above correspondence? That is the underlying question throughout this paper.

One important convex set associated with $f$ is its effective domain (1.2). Another is its supergraph $\operatorname{gph} f$, which is the convex set in $F \oplus R$ consisting of the points lying above or on the graph of $f$. Evidently, a proper convex function is positively homogeneous if and only if its supergraph is actually a convex cone. Now for each convex set there is a special convex cone, called its asymptotic cone, essentially giving the directions in which the set is infinite. In the case of gph $f$, we shall see that the asymptotic cone is itself the supergraph of a certain positively homogeneous 1.s.c. proper convex function on $F$, which we call the asymptotic function of $f$. This asymptotic function describes important growth properties of $f$. It turns out to be the support function of the closure of the effective domain of 
the conjugate $g$ of $f$. Dually, the support function of the closure of $\operatorname{dom} f$ is the asymptotic function of $g$. We shall show that this can be viewed as a limiting case of the conjugacy formulas for scalar multiples of given functions.

Each 1.s.c. proper convex function $f$ on $F$ also gives rise to a large family of level sets

$$
L_{b, \beta} f=\{x \mid f(x)+\beta \leqq(x, b)\} \text { for } b \in G \text { and } \beta \in R,
$$

each of which is a closed convex set in $F$. For any fixed $b$, the union of the $L_{h, \beta} f$ as $\beta$ ranges over $R$ is $\operatorname{dom} f$. It is clear from (1.7a) and (1.3a) that $I_{b, \beta} f$ decreases as $\beta$ increases, with

$$
g(b)=\sup \left\{\beta \in R \mid L_{h, \beta} f \neq \phi\right\} .
$$

Likewise, the conjugate function $g$ generates closed convex sets

$$
L_{a, \alpha} g=\{y \mid g(y)+\alpha \leqq(a, y)\} \text { for } a \in F \text { and } \alpha \in R,
$$

decreasing in $\alpha$ for each $a$, such that

$$
f(a)=\sup \left\{\alpha \in R \mid L_{a, \alpha} g \neq \phi\right\} .
$$

The study of these level sets will lead us to interesting results relating boundedness and continuity properties of $f$ and $g$. We shall only consider cases where $\beta<g(b)$ and $\alpha<f(a)$, so that the level sets are sure to be nonempty. The marginal cases where $\beta=g(b)$ and $\alpha=f(a)$ involve special problems treated in the theory of subdifferentials of convex functions. (See [10], [14] and the references given there.)

Our first task in analyzing these level sets will be to determine their support functions. The level sets $L_{a, a} g$ under consideration correspond one-to-one with the points $(a, \alpha)$ in $F \oplus R$ which do not belong to gph $f$. The supergraph of the support function of $L_{a, \alpha} g$ turns out to be the projecting cone of $\operatorname{gph} f$ from $(a, \alpha)$.

From the support function formulas, we shall deduce the following boundedness principle: $L_{b, \beta} f$ is bounded for all $\beta$ if and only if $b$ is an internal point of dom $g$ (i.e., along each line through $b, g$ is finite on an open segment containing $b$ ).

Starting with a locally convex Hausdorff topological vector space $E$, we can always let $F=E$ and let $G$ be the dual $E^{*}$ of $E$, with $(x, y)=y(x)$ for $y \in E^{*}$. Our results can then be applied if the topology on $G$ is taken as the weak* topology (see [1, p. 67]). Of course if $E$ is reflexive the strong topology on $E^{*}$ could also be used. Even if $E$ is not reflexive, however, our boundedness principle leads to a continuity theorem in this context. We shall see, namely, that the internal points of dom $g$ are precisely the points where the conjugate function $g$ is finite and continuous in the strong topology on the $E^{*}$. When $E$ is tonnele (in particular when $E$ is a Banach space or is reflexive, see [1, p. 2 and p. 89]), there is a dual theorem: points where $f$ is finite and continuous correspond to weak* compact level sets of $g$. 
The last result also follows from recent independent work of Moreau. In [10], which appeared shortly after our paper was submitted for publication, Moreau proved that the points where $f$ is finite and continuous in the Mackey topology $\tau(F, G)$ correspond to the level sets of $g$ which are compact in the weak topology $\sigma(G, F)$. In a tonnelé space, of course, the given topology coincides with the Mackey topology induced by the dual space. Moreau's method of proof could also be used for a more direct derivation of our theorem on boundedness, without employing the formulas for the support functions of level sets.

We also want to mention along these lines the recently published theorem of Fan [5] about polar convex sets. It is closely related to the case of Moreau's theorem where $f$ is the support function of a convex set in $G$.

2. Convex cones associated with convex sets. For each nonempty convex set $C$ in $F$ and each $a \in F$, the set

$$
P_{a} C=\operatorname{cl}\{\lambda(x-a) \mid \lambda>0, x \in C\},
$$

where "cl" denotes closure, is the projecting cone of $C$ relative to $a$. It is the smallest closed convex cone containing the translated convex set $C-a$. (This definition differs slightly from the classical one, in which the cone is not required to be closed and its vertex is at $a$ rather than at the origin.) Another important closed convex cone associated with a nonempty convex set $C$ is its asymptotic cone $0^{+} C$, which is defined as the "limit" of $\lambda C$ as $\lambda \downarrow 0$, i.e.

$$
0^{+} C=\bigcap_{\varepsilon>0}\left[\mathrm{cl} \bigcup_{0<\lambda<\varepsilon} \lambda C\right] \text {. }
$$

We want to mention here some properties of these cones which will be invoked later in the case where $C$ is replaced by the supergraph of a convex function.

Asymptotic cones seem to have been considered first by Stoker [16]. Choquet [3] has recently studied them in infinite-dimensional spaces. The characterizations of $0+C$ listed for convenience in the following theorem are all known.

THEOREM 2A. If $C$ is a nonempty closed convex set in $F$, each of the four conditions on $x \in F$ given below is equivalent to the condition that $x \in 0+C$ :

(a) the ray $\{a+\lambda x \mid \lambda \geqq 0\}$ is contained in $C$ for every $a \in C$;

(b) there exists some $a \in C$ such that $a+\lambda x \in C$ for arbitrarily large choices of $\lambda$;

(c) $C+x \subseteq C$;

(d) $(x, y) \leqq 0$ for every $y \in G$ such that the linear function $(\cdot, y)$ is bounded above on $C$.

Proof. By definition (2.2) the condition that $x \in 0^{+} C$ can be expressed as:

(e) there exist nets of scalars $\lambda_{i}>0$ and vectors $x_{i} \in C$ such that $\lim _{i} \lambda_{i}=0$ and $\lim _{i} \lambda_{i} x_{i}=x$. 
The equivalence of this with the four conditions in the theorem will be established by a cycle of implications.

(a) implies (c): Trivial (take $\lambda=1$ ).

(c) implies (b): Fix any $a \in C$. Then $a+n x \in C$ for all positive integers $n$ by induction.

(b) implies (e): Choose any unbounded increasing sequence of $\mu_{i}>0$ such that $a+u_{i} x=x_{i} \in C$. Let $\lambda_{i}=1 / \mu_{i}$. Then $\lim _{i} \lambda_{i}=0$ and $\lim _{i} \lambda_{i} x_{i}=x$.

(c) implies (d): If $(a, y) \leqq \alpha \in R$ for all $a \in C$, then

$$
(x, y)=\lim _{i} \lambda_{i}\left(x_{i}, y\right) \leqq \alpha \cdot \lim _{i} \lambda_{i}=0 .
$$

(d) implies (a): If $(a, y) \leqq \alpha \in R$ for all $a \in C$ we have $(a+\lambda x, y) \leqq \alpha$ for all $a \in C$ and $\lambda \geqq 0$ by (d). Since a closed convex set is the intersection of the closed half-spaces containing it, this finishes the proof.

Characterization (d) says that $0+C$ is polar to the cone $\operatorname{cl}\left(\operatorname{dom} \sigma_{c}\right)$ in $G$, which might be called the barrier cone of $C$ (see [7, p. 45]).

Our scalar multiple notation for the asymptotic cone of a nonempty closed convex set $C$ fits in with various algebraic formulas in a helpful way. For example, consider the formulas

$$
\lambda_{1}\left(\lambda_{2} C\right)=\left(\lambda_{2} \lambda_{2}\right) C \text { and }\left(\lambda_{1}+\lambda_{2}\right) C=\lambda_{1} C+\lambda_{2} C,
$$

which are obvious when $\lambda_{1}>0$ and $\lambda_{2}>0$.

There are similar formulas, easily verified from Theorem $2 \mathrm{~A}$, which involve the asymptotic cone, namely $0^{+}\left(\lambda_{2} C\right)=0^{+} C, \lambda_{1}\left(0^{+} C\right)=0+C, 0^{+}\left(0^{+} C\right)=0+C$, $0^{+} C=0^{+} C+0^{+} C, \lambda_{2} C=0^{+} C+\lambda_{2} C$. We can summarize all of these by saying that (2.3) holds for all $\lambda_{1} \geqq 0^{+}$and $\lambda_{2} \geqq 0^{+}$. The notation also suggests that the set

$$
\bigcup\left\{\lambda_{1} C_{1}{ }^{-}+\cdots+\lambda_{k} C_{k} \mid \lambda_{1} \geqq 0^{+}, \cdots, \lambda_{k} \geqq 0^{+}, \lambda_{1}+\cdots+\lambda_{k}=1\right\}
$$

ought to be more important in some contexts than the mere convex hull of given closed convex sets $C_{1}, \cdots, C_{k}$ (which is what (2.4) would be if the result of multiplying by zero were interpreted as $0 C_{i}=\{0\}$ instead of $0+C_{i}$ ). As a matter of fact, Choquet [3] has shown that (2.4) often gives the closed convex hull of $C_{1}, \cdots, C_{k}$.

The following projecting cone formula, which also benefits from the $0^{+}$notation is already known. Since it will be crucial in this paper, a proof is included for, completeness.

Theorem 2B (ChOQuet [3]). If $C$ is a nonempty closed convex set in $F$ and $a \notin C$, then

$$
P_{a} C=\bigcup\left\{\lambda[C-a] \mid \lambda \geqq 0^{+}\right\} .
$$

Proof. Let $K$ denote the union of the right. It is clear from definition (2.1) that $\mathrm{cl} K \supseteq P_{a} C$. Furthermore, $P_{a} C$ is closed and $P_{a} C \supseteq \lambda[C-a]$ for all $\lambda>0$, 
so $P_{a} C \supseteq 0+[C-a]$ from definition (2.2). Thus $P_{a} C \supseteq K$. It remains to show $K \supseteq \operatorname{cl} K$. If $x \in \mathrm{cl} K$, we can choose nets such that $x=\lim _{i} z_{i}$ where $z_{i} \in \lambda_{i}[C-a]$, $\lambda \geqq 0^{+}$. Taking a subnet if necessary we can suppose the $\lambda_{i}$ are all strictly positive, for otherwise $x \in 0^{+}[C-a] \subseteq K$ trivially because $0^{+}[C-a]$ is closed. Set, $z_{i}=\lambda_{i} x_{i}$, where $x_{i} \in C-a$. Since $a \notin C$ and $C$ is a closed convex set, there exists by a standard separation theorem some $b \in G$ such that $(z-a, b) \geqq 1$ for all $z \in C$. Hence

$$
\lim \sup _{i} \lambda_{i} \leqq \lim _{i} \lambda_{i}\left(x_{i}, b\right)=(x, b)<\infty .
$$

We may suppose therefore that $\lim _{i} \lambda_{i}=\lambda$, where $0 \leqq \lambda<\infty$. If $\lambda>0$, we have $(1 / \lambda)=\lim _{i} x_{i} \in C-a$, so $x \in \lambda[C-a]$. If $\lambda=0, x \in 0^{+}[C-a]$ by definition. Thus $x \in K$ in both cases, so $\mathrm{cl} K \subseteq K$.

RemarK. The asymptotic cone and projecting cones of a nonempty closed convex set $C$ were defined using the closure operation. But the characterization of $0^{+} C$ in Theorem $2 \mathrm{~A}(\mathrm{c})$, and the formula for $P_{a} C$ in Theorem 2B when $a \notin C$, show that these cones could also be defined algebraically. They do not depend on the particular topology, except to the extent that the topology has to be one in which $C$ is closed.

3. Asymptotic functions and scalar multiplication. There is a natural one-to-one correspondence between extended-real-valued functions $f$ on $F$ and their supergraphs

$$
\operatorname{gph} f=\{\langle x, \mu\rangle \mid x \in F, f(x) \leqq \mu \in R\}
$$

in $F \oplus R$. For the case of interest here, the correspondence is characterized in the following lemma, whose proof is straightforward and will be omitted.

LEMma 3A. In order that a subset $S$ of $F \oplus R$ be of the form $\operatorname{gph} f$ for some (unique) l.s.c. proper convex function $f$ on $F$, it is necessary and sufficient that $S$ be a nonempty closed convex set with $\langle 0,1\rangle \in 0^{+} S$ and $-\langle 0,1\rangle \notin 0^{+} S$.

For the rest of this section let $f$ be l.s.c. proper convex on $F$, and let $g$ be its conjugate on $G$. In view of Lemma $3 \mathrm{~A}$, we can define nonnegative right scalar multiples $f \lambda$ of $f$, all of which are again l.s.c. proper convex, by the geo metric formula

$$
\operatorname{gph}(f \lambda)=\lambda \operatorname{gph} f \text { for } \lambda \geqq 0^{+} .
$$

Evidently,

$$
(f \lambda)(x)=\lambda f((1 / \lambda) x) \text { for } \lambda>0 .
$$

We shall call $f 0^{+}$the asymptotic function of $f$. Its properties will now be described.

THEOREM 3B. Each of the following conditions on $x \in F$ and $\mu \in R$ is equivalent to the condition that $\left(f 0^{+}\right)(x) \leqq \mu$ : 
(a) $f(a+\lambda x) \leqq f(a)+\lambda \mu$ for all $a \in F$ and $\lambda \geqq 0$;

(b) there exists some $a \in \operatorname{dom} f$ such that $f(a+\lambda x) \leqq f(a)+\lambda \mu$ for arbitrarily large choices of $\lambda$;

(c) $f(z+x)-f(z) \leqq \mu$ for all $z \in \operatorname{dom} f$;

(d) $(x, y) \leqq \mu$ for all $y \in G$ with $g(y)<\infty$;

(e) there exist directed nets of vectors $x_{i} \in F$ and $\lambda_{i}>0$ such that $\lim _{i} \lambda_{i}=0$, $\lim _{i} \lambda_{i} x_{i}=x$ and $\lim _{i} \lambda_{i} f\left(x_{i}\right) \leqq \mu$.

Proof. By definition (3.2) of $f 0^{+}$, we have $\left(f 0^{+}\right)(x) \leqq \mu$ if and only if $\langle x, \mu\rangle$ is in the asymptotic cone of the set gph $f$ in (3.1). Conditions (a), (b), (c) and (e) are easily derived from the corresponding conditions in Theorem $2 \mathrm{~A}$. Condition (d) could also be derived from the earlier (d), but it is simpler to show it is equivalent to the present (c). If (c) holds, we actually have $-f(z) \leqq \mu-f(z+x)$ for all $z \in F$, so by (1.3a)

$$
\begin{aligned}
g(y) & =\sup _{z}\{(z, y)-f(z)\} \leqq \sup _{z}\{(z, y)+\mu-f(z+x)\} \\
& =\mu-(x, y)+\sup _{z}\{(z+x, y)-f(z+x)\}=\mu-(x, y)+g(y) .
\end{aligned}
$$

Hence $\mu-(x, y) \geqq 0$ whenever $g(y)<\infty$. On the other hand, if (d) holds, we see from $(1.3 b)$ that

$$
\begin{aligned}
f(z+x) & =\sup _{y}\{(z+x, y)-g(y)\} \\
& \leqq \sup \{(x, y) \mid g(y)<\infty\}+\sup \{(z, y)-g(y) \mid g(y)<\infty\} \\
& \leqq \mu+f(z)
\end{aligned}
$$

for all $x \in F$. Thus (c) holds in this case.

COROLLARY 3C. The asymptotic function of $f$ can be determined from any of the following formulas:

$$
\begin{aligned}
\left(f 0^{+}\right)(x) & =\sup _{\lambda>0}[f(a+\lambda x)-f(a)] / \lambda \text { for any } a \in \operatorname{dom} f ; \\
\left(f 0^{+}\right)(x) & =\lim _{\lambda \rightarrow \infty}[f(a+\lambda x)-f(a)] / \lambda \text { for any } a \in \operatorname{dom} f ; \\
\left(f 0^{+}\right)(x) & =\sup \{f(z+x)-f(z) \mid z \in \operatorname{dom} f\} ; \\
\left(f 0^{+}\right)(x) & =\sup \{(x, y) \mid g(y)<\infty\} ; \\
\left(f 0^{+}\right)(x) & =\lim \inf \left\{\lambda_{i} f\left(x_{i}\right) \mid \lambda_{i}>0, \lambda_{i} \rightarrow 0, \lambda_{i} x_{i} \rightarrow x\right\} \\
& =\lim \inf \left\{\left(f \lambda_{i}\right)\left(z_{i}\right) \mid \lambda_{l}>0, \lambda_{i} \rightarrow 0, z_{i} \rightarrow x\right\} .
\end{aligned}
$$

Proof. (a), (c), (d) and (e) are immediate from the conditions in 3B; (b) is equivalent to (a), because the difference quotient is a nondecreasing function of $\lambda>0$ (e.g. see [4]). 
Coroliary 3D. $\mathrm{fO}^{+}$is positively homogeneous; in fact it is the support function of the nonempty closed convex set $\mathrm{cl}(\mathrm{dom} g)$ in $G$.

Proof. This is the essence of $3 \mathrm{C}(\mathrm{d})$; cf. Example $1 \mathrm{~A}$.

Properties of asymptotic functions reduce to those of asymptotic cones when $f$ is the indicator function of a closed convex set $C$. Indeed,

$$
(f \lambda)(x)=\delta(x \mid \lambda C) \text { for all } \lambda \geqq 0^{+} \text {if } f(x)=\delta(x \mid C) .
$$

It is natural to define left scalar multiples $\lambda f$ of $f$ for $\lambda \geqq 0^{+}$by

$$
(\lambda f)(x)=\lambda[f(x)] \text { if } \lambda>0,\left(0^{+} f\right)(x)=\delta(x \mid \operatorname{cldom} f) .
$$

Then each $\lambda f$ is again 1.s.c. proper convex, and gph $(0+f)$ is the "limit" of gph $(\lambda f)$ as $\lambda \downarrow 0$. Left and right scalar multiplication are dual to one another in the sense of the following theorem, which combines a known elementary fact for $\lambda>0$ with $3 \mathrm{D}$ (cf. 1A).

THEOREM 3E. $\lambda f$ and $f \lambda$ are conjugate to $g \lambda$ and $\lambda g$, respectively, for all $\lambda \geqq 0^{+}$.

Another reason for our "right scalar multiple" notation is provided by the next theorem.

THEOREM 3F. Let $\phi$ be the function on $R \oplus F$ defined by: $\phi(\lambda, x)=(f \lambda)(x)$ for $\lambda>0, \phi(0, x)=\left(f 0^{+}\right)(x), \phi(\lambda, x)=\infty$ for $\lambda<0$. Then $\phi$ is l.s.c. proper convex and positively homogeneous. In fact $\phi$ is the support function of $\mathrm{gph} g$ in $G \oplus R$, if $R \oplus F$ and $G \oplus R$ are placed in duality with respect to

$$
(\langle\lambda, x\rangle,\langle y, \mu\rangle)=(x, y)-\lambda \mu .
$$

If $R \oplus F \oplus R$ and $R \oplus G \oplus R$ are placed in duality with respect to

$$
\left(\left\langle\lambda, x, \mu^{\prime}\right\rangle,\left\langle\lambda^{\prime}, y, \mu\right\rangle\right)=(x, y)-\lambda \mu-\lambda^{\prime} \mu^{\prime},
$$

and $\psi$ is the function on $R \oplus G$ constructed from $g$ as $\phi$ was from $f$, then $\operatorname{gph} \phi \subseteq R \oplus F \oplus R$ and $\mathrm{gph} \psi \subseteq R \oplus G \oplus R$ are closed convex cones polar to each other.

Proof. By (3.2) and 2B, gph $\phi$ is the smallest closed convex cone in $R \oplus F \oplus R$ containing $\{\langle 1, x, \mu\rangle \mid\langle x, \mu\rangle \in \operatorname{gph} f\}$.

Hence $\phi$ is a positively homogeneous l.s.c. proper convex function on $R \oplus F$ by the criterion in Lemma 3A. By Theorem 3E, the conjugate of $\phi$ with respect to $(3.5)$ is

$$
\begin{aligned}
\sup & \{(\langle\lambda, x\rangle,\langle y, \mu\rangle)-\phi(\lambda, x) \mid\langle\lambda, x\rangle \in R \oplus F\} \\
& =\sup \left\{\sup \{(x, y)-\lambda \mu-(f \lambda)(x) \mid x \in F\} \mid \lambda \geqq 0^{+}\right\} \\
& =\sup \left\{-\lambda \mu+(\lambda g)(y) \mid \lambda \geqq 0^{+}\right\}=\sigma(\langle y, \mu\rangle \mid \operatorname{gph} g) .
\end{aligned}
$$


Thus $\phi$ is the support function of gph $g$ (cf. 1A). In view of the dual of the first assertion in the proof, the polar of the cone gph $\psi$ with respect to (3.6) consists of all $\left\langle\lambda, x, \mu^{\prime}\right\rangle \in R \oplus F \oplus R$ such that

$$
0 \geqq\left(\left\langle\lambda, x, \mu^{\prime}\right\rangle,\langle 1, y, \mu\rangle\right)=(\langle\lambda, x\rangle,\langle y, \mu\rangle)-\mu^{\prime}
$$

for all $\langle y, \mu\rangle \in \operatorname{gph} g$. Thus it consists of all $\left\langle\lambda, x, \mu^{\prime}\right\rangle$ with

$$
\mu^{\prime} \geqq \sigma(\langle\lambda, x\rangle \mid \operatorname{gph} g)=\phi(\lambda, x), \text { i.e. it is gph } \phi .
$$

REMARK. The correspondences in Theorem $3 \mathrm{~F}$ were noted by Fenchel, and they apparently helped motivate his original definition of the conjugate correspondence (cf. [6] and [7, p. $88 \mathrm{ff}$.$] ). Our only contribution here has been to formalize and$ complete Fenchel's observations in terms of asymptotic functions.

4. Support functions of level sets. Just as the asymptotic function of a 1.s.c. proper convex $f$ on $F$ was defined geometrically by means of the asymptotic cone of the closed convex set $\operatorname{gph} f$, we can construct other functions from $f$ considering the projecting cones of $\operatorname{gph} f$ relative to various points $\langle a, \alpha\rangle$ of $E \oplus R$. These will turn out to be the support functions of the level sets of the conjugate $g$ of $f$, just as $f 0^{+}$, was the support function of the union of these sets, dom $g$.

Indeed, in view of Theorem $2 \mathrm{~B}$ and Lemma $3 \mathrm{~A}$, we can define a positively homogeneous 1.s.c. proper convex function $P_{a, \alpha} f$ on $F$ for each $\langle a, \alpha\rangle \notin \operatorname{gph} f$ by the formula

$$
\operatorname{gph}\left(P_{a, \alpha} f\right)=P_{\langle a, \alpha)} \operatorname{gph} f=\bigcup\left\{\lambda[(\operatorname{gph} f)-\langle a, \alpha\rangle] \mid \lambda \geqq 0^{+}\right\} .
$$

Of course, $\langle a, \alpha\rangle \notin \operatorname{gph} f$ if and only if $\alpha<f(a)$, and in this case the closed convex level set $L_{a, \alpha} g$ is sure to be nonempty (see (1.7b) and (1.8b)).

THEOREM 4A. The function $P_{a, \alpha} f$ satisfies the following formulas (where $a \in F$ and $\alpha<f(a))$ :

(a) $\left(P_{a, \alpha} f\right)(x)=\min \left\{(h \lambda)(x) \mid \lambda \geqq 0^{+}\right\}$with $h(x)=f(a+x)-\alpha$;

(b) $\left(P_{a, \alpha} f\right)(x)=\inf \{[f(a+\lambda x)-f(a)+\varepsilon] / \lambda \mid \lambda>0\}$, where $\varepsilon=f(\mathrm{a})-\alpha$, provided that $f(a)<\infty$;

(c) $\left(P_{a, \alpha} f\right)(x)=\sup \{(x, y) \mid g(y) \leqq(a, y)-\alpha\}$, i.e. $P_{a, \alpha} f$ is the support function of the level set $L_{a, \alpha} g$.

Proof. Formula (a) follows from (4.1), because $\lambda[(\operatorname{gph} f)-\langle a, \alpha\rangle]=\operatorname{gph}(h \lambda)$ for all $\lambda \geqq 0^{+}$by the definitions. If $\infty>\varepsilon=f(a)-\alpha=h(0)$, we have

$$
\left(h 0^{+}\right)(x)=\lim _{\lambda \rightarrow \infty}[h(\lambda x)-h(0)] / \lambda=\lim _{\lambda \rightarrow \infty}[f(a+\lambda x)-f(a)+\varepsilon] / \lambda
$$

by $3 \mathrm{C}(\mathrm{b})$. On the other hand,

$$
[h(1 / \lambda)](x)=h(\lambda x) / \lambda=[f(a+\lambda x)-f(a)+\varepsilon] / \lambda \text { for } 0<\lambda<\infty
$$


by the definition of right scalar multiplication. Therefore (a) implies (b) when $f(a)<\infty$. We can also calculate the conjugate of $P_{a, \alpha} f$ from (a) and $3 \mathrm{E}$ as

$$
\begin{aligned}
& \sup \left\{(x, y)-\min \left\{(h \lambda)(x) \mid \lambda \geqq 0^{+}\right\} \mid x \in F\right\} \\
& \quad=\sup \left\{\sup \{(x, y)-(h \lambda)(x) \mid x \in F\} \mid \lambda \geqq 0^{+}\right\} \\
& =\sup \left\{(\lambda k)(y) \mid \lambda \geqq 0^{+}\right\}=\delta(y \mid k(y) \leqq 0),
\end{aligned}
$$

where $k$ denotes the conjugate of $h$. Since

$$
k(y)=\sup \{(x, y)-[f(a+x)-\alpha] \mid x \in F\}=g(y)-(a, y)+\alpha,
$$

we have $k(y) \leqq 0$ if and only if $g(y) \leqq(a, y)-\alpha$, i.e. $y \in L_{a, a}$. Thus the conjugate of $P_{a, \alpha} f$ is the indicator function of $L_{a, \alpha} g$, so that we must have $\left(P_{a, \alpha} f\right)(x)$ $=\sigma\left(x \mid L_{a, \alpha} g\right)$ as in $1 \mathrm{~A}$.

COROLlary 4B. If $b \in G$ and $\inf f<0$, then

$$
\sup \{(x, b) \mid f(x) \leqq 0\}=\min \left\{(g \lambda)(b) \mid \lambda \geqq 0^{+}\right\} .
$$

If also $\inf f>-\infty$, then the right side of (4.2) can be replaced by $\inf \{g(\lambda y) / \lambda \mid \lambda>0\}$.

Proof. By the dual of $4 \mathrm{~A}$, all of these expressions give $\left(P_{0,0} g\right)(b)$.

We shall now prove the boundedness principle mentioned in the introduction.

THEOREM $4 C$. Let $b \in G$ and $\beta<g(b)$. Then the (nonempty) level set $L_{b, \beta} f$ is bounded in $F$ if and only if $b$ is an internal point of dom $g$.

Proof. A nonempty closed convex subset of $F$ is bounded in the given topology, if and only if it is bounded in the weak topology $G$ induces on $F$ (see $[1$, p. 70]), i.e. its support function is finite throughout $G$. Thus, by the dual of $4 \mathrm{~A}, L_{b, \beta} f$ is bounded if and only if

$$
G=\operatorname{dom}\left(P_{b, \beta} g\right)=\bigcup\left\{\operatorname{dom}(k \lambda) \mid \lambda \geqq 0^{+}\right\},
$$

where $k(y)=g(b+y)-\beta$. On the other hand, $b$ is an internal point of dom $g$ if and only if

$$
G=\bigcup\{\lambda[(\operatorname{dom} g)-b] \mid \lambda>0\}=\bigcup\{\lambda \operatorname{dom} k \mid \lambda>0\} .
$$

Since by definition of right scalar multiplication

$$
\operatorname{dom}(k \lambda)=\lambda \operatorname{dom} k \text { for } \lambda>0,
$$

we know (4.4) implies (4.3). Now assume (4.3) holds. For any $z \in \mathrm{dom} k$, select $\lambda \geqq 0^{+}$such that $-z \in \operatorname{dom}(k \lambda)$. If $\lambda>0$, the line segment connecting $z$ and $(-1 / \lambda) z$ lies entirely in the convex set $\operatorname{dom} k$, and contains 0 . If $\lambda=0^{+}$, the half-line $\{z+\mu(-z) \mid \mu \geqq 0\}$ lies entirely in dom $k$, because 


$$
\infty>\left(k 0^{+}\right)(-z)=\sup \{[k(z+\mu(-z))-k(z)] / \mu \mid \mu>0\}
$$

by $3 \mathrm{C}(\mathrm{a})$; this half line again contains 0 . At all events, we therefore have $0 \in \operatorname{dom} k$, i.e. $\mathrm{g}(b)<\infty$. It now follows from the dual of $4 \mathrm{~A}(\mathrm{~b})$ that

$$
\left(P_{b, \beta} g\right)(y)=\inf _{\mu>0}[g(b+\mu y)-\beta] / \mu=\inf _{\lambda>0}(k \lambda)(y) .
$$

Thus dom $\left(\mathrm{kO}^{+}\right)$can be omitted from the union in (4.3), so that (4.3) implies (4.4) by (4.5).

COROLLARY 4D. If $L_{b, \beta}$ fis bounded for some $\beta<g(b)$, then it is bounded for every $\beta \in R$.

5. Properties of effective domains. In this section we shall dualize some properties of the effective domains of a conjugate pair of functions $f$ and $g$.

First we shall generalize a fact noted by Phelps [11]. Suppose $C \subseteq F$ and $D \subseteq G$ are convex sets polar to one another. Let $f=\sigma_{D}$ and $g=\delta_{D}$ as in Example 1A. We have $C=L_{0,-1} f$ (this is the definition of polarity), and it is apparent that $f$ can also be viewed as the gauge function of $C$. Phelps proved that a point $b \in D$ is an extreme point of $D$, (i.e. $b$ does not belong to any open line segment lying in $D$ ) if and only if the convex set of differences $C_{b}-C_{b}$ is dense in $F$, where

$$
C_{b}=\left\{x \in F \mid \sigma_{D}(x) \leqq(x, b)+1\right\}=L_{b,-1} f .
$$

Of course $D=$ dom $g$ here, and $-1<g(b)=0$. The following theorem shows that a similar result is valid for any conjugate $f$ and $g$.

THeOREM 5A. Let $b \in \operatorname{dom} g$ and $\beta<g(b)$. Then $b$ is an extreme point of $\operatorname{dom} g$ in $G$ if and only if $L_{b, \beta} f-L_{b, \beta} f$ is dense in $F$.

Proof. Let $L$ be the closure of $L_{b, \beta} f-L_{b, \beta} f$, which is a nonempty convex set in $F$. We have

$$
\begin{aligned}
\sigma(y \mid L) & =\sup \left\{\left(x_{1}-x_{2}, y\right) \mid x_{1} \in L_{b, \beta} f, x_{2} \in L_{b, \beta} f\right\} \\
& =\sigma\left(y \mid L_{b, \beta} f\right)+\sigma\left(-y \mid L_{b, \beta} f\right)=\left(P_{b, \beta} g\right)(y)+\left(P_{b, \beta} g\right)(-y)
\end{aligned}
$$

by the dual of $4 \mathrm{~A}$. Since the correspondence beetween closed convex sets of their support functions is one-to-one, we conclude that $L=F$ if and only if the last expression in (5.1) equals $+\infty$ for all $y \neq 0$. But

$$
\left(P_{b, \beta} g\right)( \pm y)=\inf \{[g(b \pm \lambda y)-\beta] / \lambda \mid \lambda>0\}
$$

by the dual of $4 \mathrm{~A}(\mathrm{~b})$, inasmuch as $g(b)<\infty$ by hypothesis. Thus

$$
P_{b, \beta}(y)+P_{b, \beta}(-y)<\infty
$$

for some $y \neq 0$ if and only if $b$ is the midpoint of an open line segment between some $b-\lambda y \in \operatorname{dom} g$ and $b+\lambda y \in \operatorname{dom} g$, i.e., $b$ is not an extreme point. 
The next theorem answers the question of when the conjugate of a given function is finite everywhere.

THEOREM 5B. dom $g$ is dense in $G$ if and only if

$$
\left(f 0^{+}\right)(x)=\infty \text { for all } x \neq 0 .
$$

One actually has dom $g=G$, if and only if

$$
L_{b, \beta} f \text { is bounded for all } b \in E \text { and } \beta \in R \text {. }
$$

Proof. dom $g$ is dense if and only if $0^{+} g$ is identically zero, and this is equivalent to (5.2) because $f 0^{+}$is the conjugate of $0^{+} g$ by $3 \mathrm{E}$. We observe next that dom $g=G$ if and only if every $b \in G$ is an internal point of dom $g$. By Theorem $4 C$, this is equivalent to $L_{b, \beta} f$ being bounded whenever $\beta<g(b)$, which is trivially the same as (5.3).

COROLlaky 5C. If $F$ is finite-dimensional, conditions (5.2) and (5.3) are equivalent.

Proof. A convex set dense in a finite-dimensional space must itself be the whole space.

6. Asymptotic and projecting cones of level sets. Throughout this section it is still assumed that $f$ and $g$ are 1.s.c. proper convex functions on $F$ and $G$ conjugate to each other.

THEOREM 6A. For each $b \in G$ and $\beta<g(b)$, the asymptotic cone $0+L_{b, \beta} f$ and the projecting cone $P_{b}(\operatorname{dom} g)$ are polar to each other.

Proof. According to characterization (d) in Theorem 2A, the asymptotic cone of a nonempty closed convex set $C$ in $F$ is polar to the closure of the effective domain of the support function of $C$ in $G$. The support function of $C=L_{b, \beta} f$ is $P_{b, \beta} g$ by the dual of $4 \mathrm{~A}(\mathrm{c})$. The closure of the effective domain of $P_{b, \beta} g$ is obviously the closed convex cone generated by $(\operatorname{dom} g)-b$, i.e. it is $P_{b}(\operatorname{dom} g)$.

COROLlaRY 6B. For each $b \in G$, all the nonempty level sets of the form $L_{b, \beta} f$ have the same asymptotic cone.

Proof. The theorem trivially implies $0+L_{b, \beta} f$ is the same for all $\beta<g(b)$. This cone can be described as the set of $x$ such that $f(z+\lambda x)-(z+\lambda x, b)$ is a nonincreasing function of $\lambda$ for every $z$. If $L_{b, \beta} f$ happens to be nonempty for $\beta=g(b)$, its asymptotic cone includes these vectors, too. On the other hand, its asymptotic cone must be contained in the asymptotic cone of the other level sets, which are larger.

COROllary 6C. The set of vectors $x$ such that 


$$
f(z+x) \leqq f(z) \text { for all } z \in F
$$

is a closed convex cone in $F$ whose polar is the smallest closed convex cone in $\boldsymbol{G}$ containing dom $g$.

Proof. The set of vectors $x$ satisfying (6.1) is the common asymptotic cone of the level sets $L_{0, \beta}$ for $\beta<g(0)$, according to characterization (c) in Theorem $2 \mathrm{~A}$.

The fact that the vectors satisfying (6.1) form a closed convex cone was used extensively by the author in [13].

TheOREM 6D. Given any level set $L_{b, \beta} f$, where $\beta<g(b)$, and any point $a \notin L_{b, \beta} f$, let $\alpha$ be the real number such that $\alpha+\beta=(a, b)$. Then dually $\alpha<f(a)$ and $b \notin L_{a, \alpha} g$, and the projecting cones

$$
P_{a}\left(L_{b, \beta} f\right) \text { and } P_{b}\left(L_{a, \alpha} g\right)
$$

are polar to each other.

Proof. Since $a \notin L_{b, \beta} f$ if and only if $f(a)+\beta>(a, b)$, the hypothesis is selfdual. The conjugate of $P_{a, \alpha} f$ is the indicator function of $L_{a, \alpha} g$, whose effective domain is $L_{\alpha, a} g$ itself, so, by $6 \mathrm{~A}$ and $6 \mathrm{~B}, P_{b} L_{a, \alpha} g$ is the polar of the asymptotic cone of any nonempty level set of the form $L_{b, \mu}\left(P_{a, \alpha} f\right)$. Since $P_{a, a} f$ is positively homogeneous, we can take $\mu=0$. The level set in question is then a convex cone, and hence it coincides with its asymptotic cone. Thus the problem is to show that

$$
\left\{x \mid\left(P_{a, \alpha} f\right)(x) \leqq(x, b)\right\}=P_{a}\left(L_{b, \beta} f\right)
$$

Let

$$
h(x)=f(a+x)-\alpha=f(a+x)-(a, b)+\beta .
$$

By formula 4A(a) for $P_{a, \alpha} f$, the left side of (6.2) consists of the vectors $x$ such that $(h \lambda)(x) \leqq(x, b)$ for some $\lambda \geqq 0^{+}$. In view of formula $2 \mathrm{~B}$ for projecting cones, (6.2) will therefore certainly follow from proving that

$$
\{x \mid(h \lambda)(x) \leqq(x, b)\}=\lambda\left[\left(L_{b, \beta} f\right)-a\right] \text { for each } \lambda \geqq 0^{+} .
$$

If $\lambda$ is positive, both sides of (6.3) are equivalent to

$$
f(a+(1 / \lambda) x)+\beta \leqq(a+(1 / \lambda) x, b) .
$$

On the other hand, suppose that $\lambda=0^{+}$, and fix any $c \in\left(L_{b, \beta} f\right)-a$. By Theorem $2 \mathrm{~A}, x$ belongs to the right side of (6.3) if and only if the ray $\{c+\mu x \mid \mu \geqq 0\}$ is contained in $\left(L_{b, \beta} f\right)-a$, in other words

$$
f(a+c+\mu x)+\beta \leqq(a+c+\mu x, b) \text { for all } \mu \geqq 0 .
$$


But this is the same as

$$
h(c+\mu x) \leqq \mu(x, b) \text { for all } \mu \geqq 0 .
$$

in other words $\left(h 0^{+}\right)(x) \leqq(x, b)$. Thus $(6.3)$ is also true when $\lambda=0^{+}$.

Corollary 6E. If $f(0)>0>\inf f$, the closed convex cone generated by $\{x \mid f(x) \leqq 0\}$ is polar to the closed convex cone generated by $\{y \mid g(y) \leqq 0\}$.

Proof. Take $a=b=0$ and $\beta=0$, and use the fact that $g(0)=-\inf f$.

7. Boundedness and continuity. Local continuity properties will now be investigated using our main result about boundedness, Theorem $4 \mathrm{C}$. It will help us here if we change notation and look at the conjugate correspondence from another point of view.

Let $E$ be a locally convex Hausdorff topological vector space over $R$, and let $E^{*}$ be its dual, with $\left(x, x^{*}\right)$ written instead of $x^{*}(x)$. The formula

$$
f^{*}\left(x^{*}\right)=\sup \left\{\left(x, x^{*}\right)-f(x) \mid x \in E\right\} \text { for all } x^{*} \in E^{*}
$$

defines a conjugate operation which takes certain functions on $E$ into functions on $E^{*}$, just as the adjoint operation takes linear transformations $E \rightarrow E$ into transformations $E^{*} \rightarrow E^{*}$. If the operation is applied twice, one has

$$
f^{* *}\left(x^{* *}\right)=\sup \left\{\left(x^{*}, x^{* *}\right)-f^{*}\left(x^{*}\right) \mid x^{*} \in E^{*}\right\} \text { for all } x^{* *} \in E^{* *},
$$

where $E^{* *}$ is the bidual of $E$. Of course, $E$ and $E^{*}$ are in duality with respect to $\left(x, x^{*}\right)$, and the original topology on $E$ and the weak* topology on $E^{*}$ are compatible with this duality $[1$, p. 69]. Hence, the results proved in earlier sections can be applied in the new notational system, with $E=F, E^{*}=G, x^{*}=y, f^{*}=g$, etc., provided the $G$ topology is interpreted as the weak* topology. If $E$ is reflexive, the strong topology on $E^{*}$ may be used instead. For example, the basic facts about conjugacy may now be viewed as follows: The conjugates $f^{*}$ of the l.s.c proper convex functions $f$ on $E$ are precisely the weak* 1.s.c. proper convex functions on $E^{*}$. (Such functions are a fortiori strongly 1.s.c., but in the nonreflexive case there will be strongly l.s.c. proper convex functions on $E^{*}$ which are not of the form $f^{*}$.) Furthermore, the restriction of $f^{* *}$ to $E$ (considered as a subspace of $E^{* *}$ ) is $f$. We assume, of course, here and henceforth, that $f$ is 1 .s.c. proper convex on $E$.

The derived functions $f^{*} 0^{+}$and $P_{a^{*}, \alpha^{*}} f^{*}$, for $\alpha^{*}<f^{*}\left(a^{*}\right)$, do not depend on the topology being considered on $E^{*}$, since they arise algebraically from $f$ through (7.1) and formulas like those in $3 \mathrm{C}$ and $4 \mathrm{~A}$. Caution must be used with $0^{+} f^{*}$, however, because the weak* closure of a convex set in $E^{*}$ can be larger than its strong closure in the nonreflexive case. It may be deduced from $4 \mathrm{~A}(\mathrm{c})$ that

$$
\left(P_{a, \alpha} f\right)^{* *}=P_{a, \alpha}\left(f^{* *}\right) \text { when } a \in E \text { and } \alpha<f(a)=f^{* *}(a) \text {. }
$$


Thus $P_{a, \alpha} f$ is the restriction of $P_{a, \alpha}\left(f^{* *}\right)$ to $E$. It is also true that $f 0^{+}$is the restriction of $f^{* *} 0^{+}$to $E$ (see $3 \mathrm{C}(\mathrm{d})$ ), but $f^{* *} 0^{+}$is not always the same as $\left(f 0^{+}\right)^{* *}$ (c.g. when $\operatorname{dom} f^{*}$ is weak ${ }^{*}$ dense in $E^{*}$ but not strongly dense). The relationship between the level sets of $f$ and those of its "bi-conjugate" is quite simple. For each $a^{*} \in E^{*}$ and $\alpha^{*}<f^{*}\left(a^{*}\right), L_{a^{*}, \alpha^{*}}{ }^{* *}$ is the closure of $L_{a^{*}, \alpha^{*}} f$ in the weak topology on $E^{* *}$ induced by $E^{*}$. This follows from the fact that, by the appropriate versions of $4 \mathrm{~A}(\mathrm{c})$, both of these convex sets can be viewed as having the same support function $P_{a^{*}, a^{*}} f^{*}$ on $E^{*}$. (Consider first the duality between $E$ and $E^{*}$ and then that between $E^{* *}$ and $E^{*}$.)

We shall now prove a continuity theorem having many consequences. The relationship between this result and the one of Moreau [10] has already been pointed out in $\$ 1$.

THEOREM 7A.

(a) For any $\alpha^{*}<f^{*}\left(a^{*}\right), L_{a^{*}, \alpha^{*}} f$ is bounded if and only if $f^{*}$ is finite und strongly continuous at $a^{*}$.

(b) If $E$ is tonnele then, for any $\alpha<f(a), f$ is finite and continuous at $a$ if and only if $L_{\alpha, a} f^{*}$ is weak* compact.

Proof. If $f^{*}$ is finite and strongly continuous at $a^{*}$, then $a^{*}$ is an internal point of $\operatorname{dom} f^{*}$. The converse of this fact will be enough to prove (a), in view of Theorem 4C. Suppose $a^{*}$ is internal to $\operatorname{dom} f^{*}$. Since $f^{*}$ is already strongly 1.s.c. at $a^{*}$, we can verify strong continuity at $a^{*}$ by showing that, for arbitrary $\varepsilon>0$,

$$
U_{\varepsilon}^{*}=\left\{x^{*} \in E^{*} \mid f^{*}\left(a^{*}+x^{*}\right) \leqq f^{*}\left(a^{*}\right)+\varepsilon\right\}
$$

is a strong neighborhood of the origin. Now, $U_{\varepsilon}^{*}$ is the polar of the set

$$
U_{\varepsilon}=\left\{x \in E\left|\left(x, x^{*}\right) \leqq 1\right| \text { for all } x^{*} \in U_{\varepsilon}^{*}\right\},
$$

because $U_{\varepsilon}^{*}$ is a weak* closed convex subset of $E^{*}$ containing 0 (see [1, p. 52]). In addition, $U_{\varepsilon}^{*}$ is absorbent. This results from the assumption that $a^{*}$ is an internal point of $\operatorname{dom} f^{*}$, since a convex function is automatically continuous along any open line segment where it is finite (see [4]). Therefore $U_{\varepsilon}$ is weakly bounded, and hence bounded in the initial topology on $E$. Thus $U_{\varepsilon}^{*}$ is the polar of bounded set in $E$, which, by definition, means that $U_{\varepsilon}^{*}$ is a neighborhood of 0 in the strong topology on $E^{*}$.

Applying (a) to $E^{*}$ in place of $E$, we see that, for $a \in E$ and $\alpha<f^{* *}(a)=f(a)$, $L_{\alpha, a} f^{*}$ is strongly bounded in $E^{*}$ if and only if $f^{* *}$ is finite and continuous at $a$ in the strong topology on $E^{* *}$. When $E$ is tonnelé, the closed and strongly bounded convex sets in $E^{*}$ are the weak* compact ones [1, p. 65 and p. 86], and the initial topology on $E$ is the same as its relative topology as a subspace of $E^{* *}[1, \mathrm{p} .87]$. Inasmuch as $f^{* *}$ coincides with $f$ on $E$, this proves (b). 
Corollary 7B. $f^{*}$ is continuous at $a^{*}$ in the strong topology if and only if, along each line through $a^{*}$, the restriction of $f^{*}$ is continuous at $a^{*}$.

Proof. The condition is trivially necessary. Its sufficiency when $f^{*}\left(a^{*}\right)<\infty$ follows from 7A and 4C, because it implies $a^{*}$ is an internal point of $\operatorname{dom} f^{*}$. (A finite convex function on an open line segment is always continuous, as pointed out above.) When $f^{*}\left(a^{*}\right)=\infty, f^{*}$ is strongly continuous at $a^{*}$ trivially, since it is strongly l.s.c.

Corollary 7C. If E is tonnelé, $f$ is continuous at every internal point of its effective domain.

Proof. If $a$ is an internal point of $\operatorname{dom} f$, the level sets $L_{a, a} f^{*}$ are bounded by 4C. But closed bounded convex subsets of the dual of a tonnelé space are weak* compact. Thus $f$ is continuous at $a$ by $7 \mathrm{~A}(\mathrm{~b})$ when $E$ is tonnelé.

COROLLARY 7D. If $L_{a^{*}, \alpha^{*}} f$ is bounded for some $\alpha^{*}<f^{*}\left(a^{*}\right)$, then there exists a strong neighborhood $U^{*}$ of $a^{*}$ in $E^{*}$ such that $L_{b^{*}, \beta^{*}} f$ is bounded for all $b^{*} \in U^{*}$ and $\beta^{*} \in R$.

Proof. Let $U^{*}$ be the interior of $\operatorname{dom} f^{*}$ in the strong topology. The hypothesis implies that $a^{*} \in U^{*}$ by $7 \mathrm{~A}(\mathrm{a})$. Every point of $U^{*}$ is internal to $\operatorname{dom} f^{*}$, so the conclusion now follows for $b^{*} \in U^{*}$ and $\beta^{*}<f^{*}\left(b^{*}\right)$ by $4 \mathrm{C}$. The extension to the case where $\beta^{*} \geqq f^{*}\left(b^{*}\right)$ is trivial.

The first part of the next corollary has already been noted by Hörmander [8, Theorem 7].

COROllary 7E. A nonempty closed convex set $C$ in $E$ is bounded if and only if its support function is strongly continuous throughout $E^{*}$. If $E$ is tonnelé, a nonempty closed convex set $C^{*}$ in $E^{*}$ is weak* compact if and only if its support function on $E$ is continuous everywhere.

Proof. In the first case take, $f=\delta_{C}$ and $f^{*}=\sigma_{C}$ as in Example 1A and apply $7 \mathrm{~A}(\mathrm{a})$. In the second case, take $f=\sigma_{C^{*}}, f^{*}=\delta_{C^{*}}$ and apply 7A(b).

Corollary 7F. Let $K$ be a nonempty closed convex cone in $E,\{0\} \neq K \neq E$, and let $K^{*}$ be its polar in $E^{*}$. Then $-a^{*}$ belongs to the strong interior of $K^{*}$ if and only if $\left\{x \in K \mid\left(x, a^{*}\right)=1\right\}$ is nonempty and bounded. If $E$ is tonnelé, $-a$ is interior to $K$ if and only if $\left\{x^{*} \in K^{*} \mid\left(a, x^{*}\right)=1\right\}$ is nonempty and weak* compact.

Proof. Let $f=\delta_{K}$ and $f^{*}=\delta_{K^{*}}$ as in Example 1B. Then $L_{-a^{*},-1} f$ $=\left\{x \in K \mid\left(x, a^{*}\right) \leqq 1\right\}$. Since $K$ contains more than just 0 , this set is bounded if and only if $\left\{x \in K \mid\left(x, a^{*}\right)=1\right\}$ is nonempty and bounded. The first conclusion of the corollary now follows from $7 \mathrm{~A}(\mathrm{a})$. The second conclusion is derived likewise from $7 \mathrm{~A}(\mathrm{~b})$. 
Just as boundedness of level sets is dual to continuity at points, boundedness of effective domains is dual to uniform continuity. We shall prove this now as our final result.

CoRollary 7G. $f^{*}$ is finite and uniformly strongly continuous throughout $E^{*}$ if and only if $\operatorname{dom} f$ is bounded in E. If $E$ is tonnele, then $f$ is finite and uniformly continuous throughout $E$ if and only if $\operatorname{dom} f^{*}$ is relatively weak* compact.

Proof. If $f^{*}$ is finite and uniformly strongly continuous, we must have $\operatorname{dom}\left(f^{*} 0^{+}\right)=E^{*}$ by formula $3 C(\mathrm{c})$. This implies $\operatorname{dom} f$ is weakly bounded (and hence bounded) by the dual of $3 \mathrm{C}(\mathrm{d})$. Conversely, suppose $\operatorname{dom} f$ is bounded. Then $f^{*} 0^{+}$is finite and strongly continuous on $E^{*}$ by $7 \mathrm{E}$, because it is the support function of $\operatorname{cl}(\operatorname{dom} f)$. Moreover

$$
\left|f^{*}\left(y^{*}\right)-f^{*}\left(z^{*}\right)\right| \leqq \max \left\{\left(f^{*} 0^{+}\right)\left(y^{*}-z^{*}\right),\left(f^{*} 0^{+}\right)\left(z^{*}-y^{*}\right)\right\}
$$

for all $y^{*} \in E^{*}$ and $z^{*} \in E^{*}$ by $3 C(\mathrm{c})$, so we can conclude from this that $f^{*}$ is finite and uniformly strongly continuous. The second part of the corollary is proved similarly.

REMARK. Suppose dom $f$ is bounded, and let

$$
\rho\left(x^{*}\right)=\sup \left\{\left|\left(x, x^{*}\right)\right| \mid x \in \operatorname{dom} f\right\}=\max \left\{\left(f^{*} 0^{+}\right)\left(x^{*}\right),\left(f^{*} 0^{+}\right)\left(-x^{*}\right)\right\}
$$

(see $3 \mathrm{C}(\mathrm{d})$ ). Then $\rho$ is a strongly continuous seminorm on $E^{*}$ by definition of the strong topology. According to the proof above, we have

$$
\left|f^{*}\left(y^{*}\right)-f^{*}\left(z^{*}\right)\right| \leqq \rho\left(y^{*}-z^{*}\right) \text { for all } y^{*} \in E^{*} \text { and } z^{*} \in E^{*}
$$

In fact $\rho$ is the smallest function with this property, because

$$
\rho\left(x^{*}\right)=\sup \left\{\left|f^{*}\left(y^{*}\right)-f^{*}\left(z^{*}\right)\right| \mid y^{*}-z^{*}=x^{*}\right\}
$$

by formula $3 \mathrm{C}(\mathrm{c})$ for $f^{*} 0^{+}$. If $E$ is a normed linear space, it is immediate from these facts that

$$
\sup \left\{\left|f^{*}\left(y^{*}\right)-f^{*}\left(z^{*}\right)\right| /\left\|y^{*}-z^{*}\right\| \mid y^{*} \neq z^{*}\right\}=\sup \{\|x\| \mid x \in \operatorname{dom} f\} .
$$

\section{REFERENCES}

1. N. Bourbaki, Espaces vectoriels topologiques, Chapters III, IV, and V, Hermann, Paris, 1955.

2. A. Brøndsted, Conjugate convex functions in topological vector spaces, Mat.-Fys. Medd. Danske Vid. Selsk. 34 (1964).

3. G. Choquet, Ensembles et cônes convexes faiblement complets, C. R. Acad. Sci. Paris 254 (1962), 1908-1910.

4. H. G. Eggleston, Convexity, Cambridge Univ. Press, New York, 1958.

5. K. Fan, A generalization of the Alaoglu-Bourbaki theorem and its applications, Math. Z. 88 (1965), 48-60.

6. W. Fenchel, On conjugate convex functions, Canad. J. Math. 1 (1949), 73-77. 
7. - Convex cones, sets and functions, Lecture notes, mimeograph, Princeton Univ. Princeton, N. J., 1953.

8. L. Hörmander, Sur la fonction d'appui des ensembles convexes, dans une espace localement convexe, Ark. Mat. 3 (1954), 181-186.

9. J. J. Moreau, Fonctions convexes en dualité, (multigraph), Faculté des Sciences, Séminaires de Mathématiques, Université de Monpellier, Montpellier, 1962.

10. - Sur la polaire d'une fonctionelle semicontinue supérieurment, C. R. Acad. Sci. Paris 258 (1964), 1128-1130.

11. R. R. Phelps, Extreme points of polar convex sets, Proc. Amer. Math. Soc. 12 (1961), 291-296.

12. R. T. Rockafellar, Convex functions and dual extremum problems, Doctoral dissertation, Harvard University, Cambridge, Mass., 1963.

13. - Helly's theorem and minima of convex functions, Duke Math. J. 32 (1965), 381-398.

14. - Extension of Fenchel's duality theorem for convex functions, Duke Math. J. 33 (1966), 81-90.

15. - Dual extremum problems involving convex functions, Pacific J. Math. (1966) (to appear).

16. J. J. Stoker, Unbounded convex point sets, Amer. J. Math. 62 (1940), 165-179.

The University OF TeXas,

Austin, Texas 